\title{
Substance-Induced Persisting Amnestic Disorder
}

National Cancer Institute

\section{Source}

National Cancer Institute. Substance-Induced Persisting Amnestic Disorder. NCI

Thesaurus. Code 192643.

A disorder involving memory impairment (either anterograde or retrograde) that is etiologically linked to the persisting effects of substance use. The memory impairment persists beyond the usual duration of substance intoxication or withdrawal. 